\title{
Variação de entropia na termalização espontânea de dois blocos distintos revisitada
}

\author{
Change of entropy in the spontaneous thermalization of two distinct blocks revisited
}

\author{
Wilson Hugo C. Freire*1, Daniela Leite ${ }^{2}$ \\ ${ }^{1}$ Departamento de Física, Universidade Regional do Cariri, Juazeiro do Norte, CE, Brasil \\ ${ }^{2}$ Departamento de Física, Universidade Regional do Cariri, Campus Crajubar, \\ Juazeiro do Norte, CE, Brasil
}

Recebido em 10 de setembro de 2015. Aceito em 5 de dezembro de 2015

\begin{abstract}
Em um artigo recente (Revista Brasileira de Ensino de Física, primeira referência dessa nota), F. Lima apresentou uma expressão para a variação de entropia de um sistema fechado constituído de dois blocos distintos no processo de termalização que ocorre após os blocos, inicialmente com temperaturas diferentes, serem colocados em contato térmico. Com isso ele mostrou, usando cálculo diferencial, que esta variação de entropia é positiva, em pleno acordo com a versão entrópica da segunda lei da termodinâmica. Nesta nota apresentamos uma demonstração alternativa. Inicialmente deduzimos, num nível elementar, o estado final de equilíbrio térmico do sistema a partir da nulidade da derivada primeira da entropia. Em seguida mostramos que a entropia deste sistema possui caráter crescente e verificamos, a partir da derivada segunda, que o estado final de equilíbrio é um estado de máxima entropia e, portanto, temos uma variação positiva de entropia.
\end{abstract}

Palavras-chave: equilíbrio térmico, variação de entropia, segunda lei da termodinâmica.

In a recent article (Revista Brasileira de Ensino de Física, first refrence of this note), F. Lima presented an expression for the change of entropy of a closed system constituted by two distinct blocks in the thermalization process which occurs when the blocks, initially with different temperatures, are put in thermic contact. From this he showed, using differential calculus, that this change of entropy is positive, in full agreement with the entropic version of the second law of thermodynamics. In this note we presented an alternative demonstration. Initially we deduced, in an elementary level, the final state of thermal equilibrium of system from nullity of the first derivative of entropy. Then we showed that the entropy of the system is increasing. Finally we showed, from the second derivative of entropy, that the final equilibrium state of system is a state of maximum entropy and therefore we have a positive change of entropy.

Keywords: thermic equilibrium, change of entropy, second law of thermodynamics..

Vamos considerar um sistema isolado composto de dois blocos ou subsistemas $\mathbf{A}$ e $\mathbf{B}$ com capacidades térmicas constantes $C_{\mathbf{A}}$ e $C_{\mathbf{B}}$, respectivamente, separados inicialmente por uma parede adiabática $\mathbf{P}$, conforme a Fig. 1. Vamos supor que o sistema se encontra inicialmente no estado $i=\left(T_{\mathbf{A} i}, T_{\mathbf{B} i}\right)$ onde $T_{\mathbf{A} i}$ e $T_{\mathbf{B} i}$ são respectivamente as temperaturas absolutas iniciais dos blocos A e $\mathbf{B}$. Sem perda de generalidade, podemos considerar $T_{\mathbf{B} i}>T_{\mathbf{A} i}>0$.

*Endereço de correspondência: wilsonhugo1@gmail.com
Se removermos a parede $\mathbf{P}$ de modo que os blocos passem a ficar em contato térmico vamos mostrar de um modo simples que o estado final $f=\left(T_{\mathbf{A} f}, T_{\mathbf{B} f}\right)$ é um estado de equilíbrio térmico entre os blocos, ou seja, $T_{\mathbf{A} f}=T_{\mathbf{B} f}$. Notemos primeiramente que, como se trata de um sistema isolado, pela conservação de energia temos (vide Ref. [1], por exemplo)

$$
\begin{aligned}
& Q_{\mathbf{A}}+Q_{\mathbf{B}}=C_{\mathbf{A}}\left(T_{\mathbf{A} f}-T_{\mathbf{A} i}\right)+ \\
& C_{\mathbf{B}}\left(T_{\mathbf{B} f}-T_{\mathbf{B} i}\right)=0 \\
& \therefore \quad T_{\mathbf{B} f}=T_{\mathbf{B} i}-\frac{C_{\mathbf{A}}}{C_{\mathbf{B}}}\left(T_{\mathbf{A} f}-T_{\mathbf{A} i}\right) .
\end{aligned}
$$




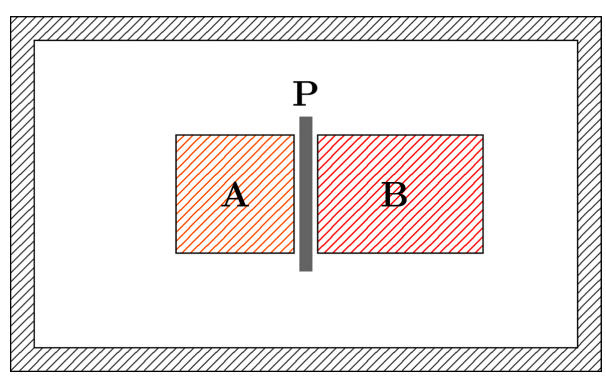

Figura 1: Sistema fechado composto de dois blocos distintos.

Não é necessário supor que o bloco $\mathbf{B}$, de maior temperatura inicial, cede calor ao bloco $\mathbf{A}$ : vamos deduzir isto mais adiante.

A variação de entropia do sistema no processo irreversível $\left(T_{\mathbf{A} i}, T_{\mathbf{B} i}\right) \rightsquigarrow\left(T_{\mathbf{A} f}, T_{\mathbf{B} f}\right)$ deveria ser calculada escolhendo um processo reversível entre os estados inicial e final do processo em consideração. Este é o procedimento padrão apresentado, por exemplo, nas Seções 20.3 da Ref. [2], 10.8 da Ref. [3], 2.2 da Ref. [4] e 11 da Ref. [5]. Entretanto, conforme apontado por Lima na Ref. [1] e, mais recentemente, na Ref. [6], não podemos tomar diretamente um processo reversível entre os estados $\left(T_{\mathbf{A} i}, T_{\mathbf{B} i}\right)$ e $\left(T_{\mathbf{A} f}, T_{\mathbf{B} f}\right)$, pois o estado inicial $\left(T_{\mathbf{A} i}, T_{\mathbf{B} i}\right)$, após a retirada da parede $\mathbf{P}$, não é um estado de equilíbrio. Para contornar esta dificuldade seguimos a mesma linha de raciocínio utilizada por Lima em [1] e [6], onde os blocos são analisados separadamente. Vamos imaginar um processo em que o bloco $\mathbf{A}$ é colocado sussecivamente em contato com um grande número $N$ de reservatórios cujas temperaturas variam de $T_{\mathbf{A} i}$ para $T_{\mathbf{A} f}$ fazendo com que o bloco $\mathbf{A}$ passe por uma sequência de processos quase reversíveis. Dessa forma calculamos a variação $\Delta S_{\mathrm{A}}$ do bloco $\mathbf{A}$ adicionando as contribuições $\delta Q_{\mathbf{A}} / T_{\mathbf{A}}$ para todos estes processos e tomando o limite com $N \mapsto \infty$ e $\delta Q_{\mathbf{A}} \mapsto 0$. Dessa forma, temos

$$
\begin{aligned}
& \Delta S_{\mathbf{A}}=\int_{R_{\mathbf{A}}} \frac{d Q_{\mathbf{A}}}{T_{\mathbf{A}}}= \\
& \int_{T_{\mathbf{A} i}}^{T_{\mathbf{A} f}} \frac{C_{\mathbf{A}} \cdot d T_{\mathbf{A}}}{T_{\mathbf{A}}}=C_{\mathbf{A}} \ln \left(\frac{T_{\mathbf{A} f}}{T_{\mathbf{A} i}}\right),
\end{aligned}
$$

onde $R_{\mathrm{A}}$ é o processo reversível no limite descrito anteriormente. Analogamente, a variação de entropia do bloco B é

$$
\Delta S_{\mathbf{B}}=\int_{T_{\mathbf{B} i}}^{T_{\mathbf{B} f}} \frac{C_{\mathbf{B}} \cdot d T_{\mathbf{B}}}{T_{\mathbf{B}}}=C_{\mathbf{B}} \ln \left(\frac{T_{\mathbf{B} f}}{T_{\mathbf{B} i}}\right)
$$

e, então, a variação de entropia total dos blocos é

$$
\begin{aligned}
& \Delta S_{\mathrm{sist}}=\Delta S_{\mathbf{A}}+\Delta S_{\mathbf{B}}= \\
& C_{\mathbf{A}} \ln \left(\frac{T_{\mathbf{A} f}}{T_{\mathbf{A} i}}\right)+C_{\mathbf{B}} \ln \left(\frac{T_{\mathbf{B} f}}{T_{\mathbf{B} i}}\right) .
\end{aligned}
$$

Mas com o vínculo dado pela Eq. (1) podemos escrevê-la em função da temperatura final do bloco inicialmente mais frio, ou seja, de $T_{\mathbf{A} f} \equiv T$

$$
\begin{aligned}
& \Delta S_{\text {sist }}=C_{\mathbf{A}} \ln \left(\frac{T}{T_{\mathbf{A} i}}\right)+ \\
& C_{\mathbf{B}} \ln \left(\frac{T_{\mathbf{B} i}-\left(C_{\mathbf{A}} / C_{\mathbf{B}}\right)\left(T-T_{\mathbf{A} i}\right)}{T_{\mathbf{B} i}}\right) .
\end{aligned}
$$

Assim, pondo $\Delta S_{\text {sist }}=S_{\text {sist }}(f)-S_{\text {sist }}(i)$, onde $i=\left(T_{\mathbf{A} i}, T_{\mathbf{B} i}\right)$ e $f=\left(T_{\mathbf{A} f}, T_{\mathbf{B} f}\right)=\left(T, T_{\mathbf{B} f}\right)$, temos que a entropia do sistema em relação ao estado de equilíbrio inicial pode ser escrita como

$$
\begin{gathered}
S(T)=C_{\mathbf{A}} \ln \left(\frac{T}{T_{\mathbf{A} i}}\right)+ \\
C_{\mathbf{B}} \ln \left(\frac{T_{\mathbf{B} i}-\left(C_{\mathbf{A}} / C_{\mathbf{B}}\right)\left(T-T_{\mathbf{A} i}\right)}{T_{\mathbf{B} i}}\right)+\text { constante. }
\end{gathered}
$$

Para calcular o novo estado de equilíbrio do sistema basta considerar que a entropia de um sistema isolado de influências externas se torna constante no estado final de equilíbrio, visto que a partir deste não há mais modificações macroscópicas no sistema. Logo o estado de equilíbrio final é tal que

$$
\frac{d S}{d T}=0
$$

Derivando a Eq. (6) temos

$$
\frac{d S}{d T}=C_{\mathbf{A}}\left[\frac{1}{T}-\frac{1}{T_{\mathbf{B} i}-\left(C_{\mathbf{A}} / C_{\mathbf{B}}\right)\left(T-T_{\mathbf{A} i}\right)}\right],
$$

que se anula exatamente quando

$$
T=T_{\mathbf{A} f}=T_{\mathbf{B} i}-\frac{C_{\mathbf{A}}}{C_{\mathbf{B}}}\left(T-T_{\mathbf{A} i}\right),
$$

e pela Eq. (1)

$$
T=T_{\mathbf{A} f}=T_{\mathbf{B} f},
$$

o que corresponde a situação de equilíbrio térmico entre os blocos. Fazendo então $T_{\mathbf{A} f}=T_{\mathbf{B} f} \equiv T_{\text {eq }}$ na Eq. (1) temos

$$
T_{\mathrm{eq}}=\frac{C_{\mathbf{A}} T_{\mathbf{A} i}+C_{\mathbf{B}} T_{\mathbf{B} i}}{C_{\mathbf{A}}+C_{\mathbf{B}}} .
$$

Tendo em vista que $0<T_{\mathbf{A} i}<T_{\mathbf{B} i}$ temos que: 
1. A temperatura de equilíbrio térmico é intermediaria: $T_{\mathbf{A} i}<T_{\text {eq }}<T_{\mathbf{B} i}$. De fato $T_{\text {eq }}$ é, pela Eq. (10), a média ponderada de $T_{\mathbf{A} i}$ e $T_{\mathbf{B} i}$ com pesos estatísticos $C_{\mathbf{A}} /\left(C_{\mathbf{A}}+C_{\mathbf{B}}\right)$ e $C_{\mathbf{B}} /\left(C_{\mathbf{A}}+C_{\mathbf{B}}\right)$.

2. O calor se transfere do bloco de maior temperatura inicial para o de menor temperatura inicial. De fato, considerando a Eq. (1), temos que o calor recebido pelo bloco $\mathbf{A}$, inicialmente mais frio, é $Q_{\mathbf{A}}=C_{\mathbf{A}}\left(T_{\text {eq }}-T_{\mathbf{A} i}\right)=-C_{\mathbf{B}}\left(T_{\text {eq }}-T_{\mathbf{B} i}\right)=$ $-Q_{\mathbf{B}}>0$, visto que $T_{\mathbf{A} i}<T_{\text {eq }}<T_{\mathbf{B} i}$. Assim o bloco $\mathbf{B}$, inicialmente mais quente, cede calor ao bloco $\mathbf{A}$.

Vamos agora mostrar, de duas maneiras simples, que a variação de entropia deste sistema de dois blocos no referido processo de termalização é positiva. Primeiramente notemos que, tendo em vista as Eqs. (2) e (3), a variação de entropia dos blocos pode ser colocada na forma

$$
\begin{aligned}
& \Delta S_{\mathrm{sist}}=C_{\mathbf{A}} \int_{T_{\mathbf{A} i}}^{T_{\mathrm{eq}}} F\left(T_{\mathbf{A}}\right) \cdot d T_{\mathbf{A}}+ \\
& C_{\mathbf{B}} \int_{T_{\mathbf{B} i}}^{T_{\mathrm{eq}}} F\left(T_{\mathbf{B}}\right) \cdot d T_{\mathbf{B}},
\end{aligned}
$$

onde $F(T)=1 / T$ é uma função contínua para $T>0$. Pelo teorema do valor médio para integrais (vide 7 ou [8]) existem $T_{\mathbf{A} m}$ e $T_{\mathbf{B} m}$ tais que $T_{\mathbf{A} i}<T_{\mathbf{A} m}<$ $T_{\text {eq }}<T_{\mathbf{B} m}<T_{\mathbf{B} i}$ e além disso

$$
\begin{array}{r}
\Delta S_{\mathrm{sist}}=F\left(T_{\mathbf{A} m}\right) \cdot C_{\mathbf{A}} \cdot\left(T_{\mathrm{eq}}-T_{\mathbf{A} i}\right)+ \\
F\left(T_{\mathbf{B} m}\right) \cdot C_{\mathbf{B}} \cdot\left(T_{\mathrm{eq}}-T_{\mathbf{B} i}\right) .
\end{array}
$$

Considerando a Eq. (1) e $F(T)=1 / T$, temos

$$
\Delta S_{\text {sist }}=Q_{\mathbf{A}}\left[\frac{1}{T_{\mathbf{A} m}}-\frac{1}{T_{\mathbf{B} m}}\right],
$$

em que $Q_{\mathbf{A}}=C_{\mathbf{A}}\left(T_{\text {eq }}-T_{\mathbf{A} i}\right)=-C_{\mathbf{B}}\left(T_{\text {eq }}-T_{\mathbf{B} i}\right)>0$ que é o calor recebido pelo bloco $\mathbf{A}$ inicialmente mais frio. E tendo em vista que $T_{\mathbf{A} m}<T_{\mathbf{B} m}$ segue-se que $\Delta S_{\text {sist }}>0$, conforme obtido por Lima nas Refs. [1 e [6]. Vale salientar que o resultado expresso pela Eq. (12) é obtido na Seção 25-8 da Ref. [9] de forma intuitiva sem menção explícita ao teorema do valor médio para integrais. Mas vale salientar também que este teorema, explicitado aqui, garante não apenas a existência das temperaturas médias nos intervalos em questão mas também informa qual delas é maior, o que é crucial para a determinação do sinal de $\Delta S_{\text {sist }}$ pela Eq. 12 .

Outra forma interessante de mostrar que a variação de entropia no referido processo é positiva advém do caráter crescente da entropia do sistema em consideração, o que pode ser mostrado mediante o uso da Eq. (8). De acordo com esta equação podemos mostrar que

$$
\begin{array}{r}
\frac{d S}{d T}=C_{\mathbf{A}}\left[\frac{1}{T}-\frac{1}{T_{\mathbf{B} i}-\left(C_{\mathbf{A}} / C_{\mathbf{B}}\right)\left(T-T_{\mathbf{A} i}\right)}\right]>0, \\
\text { para } T_{\mathbf{A} i} \leqslant T<T_{\text {eq }} .
\end{array}
$$

Antes de demonstrar isto matematicamente notemos (fisicamente) que quando os blocos $\mathbf{A}$ e $\mathbf{B}$ estão quase no equilíbrio térmico, a temperatura de $\mathbf{A}$ (inicialmente mais frio) é um pouco menor do que $T_{\text {eq }}$ que, por sua vez, é um pouco menor do que a temperatura de $\mathbf{B}$, ou seja, $T_{\mathbf{A}}=T \lesssim T_{\text {eq }} \lesssim T_{\mathbf{B}}=$ $T_{\mathbf{B} i}-\left(C_{\mathbf{A}} / C_{\mathbf{B}}\right)\left(T-T_{\mathbf{A} i}\right)$ e dessa forma temos, pela Eq. 13$], d S / d T>0$ para $T \lesssim T_{\text {eq. }}$. A rigor, para $T_{\mathbf{A}}=T<T_{\text {eq }}$, temos

$$
\begin{array}{r}
T_{\mathbf{B}}=T_{\mathbf{B} i}-\frac{C_{\mathbf{A}}}{C_{\mathbf{B}}}\left(T-T_{\mathbf{A} i}\right)= \\
\frac{\left(C_{\mathbf{A}} T_{\mathbf{A} i}+C_{\mathbf{B}} T_{\mathbf{B} i}\right)-C_{\mathbf{A}} T}{C_{\mathbf{B}}},
\end{array}
$$

e, pela Eq. 10 ,

$$
\begin{array}{r}
T_{\mathbf{B}}=T_{\mathbf{B} i}-\frac{C_{\mathbf{A}}}{C_{\mathbf{B}}}\left(T-T_{\mathbf{A} i}\right)=\frac{\left(C_{\mathbf{A}}+C_{\mathbf{B}}\right) T_{\mathrm{eq}}-C_{\mathbf{A}} T}{C_{\mathbf{B}}}= \\
\frac{C_{\mathbf{A}}\left(T_{\mathrm{eq}}-T\right)+C_{\mathbf{B}} T_{\mathrm{eq}}}{C_{\mathbf{B}}}>\frac{C_{\mathbf{B}} T_{\mathrm{eq}}}{C_{\mathbf{B}}}=T_{\mathrm{eq}}>T .
\end{array}
$$

Assim, pela Eq. 13, temos $d S / d T>0$ para $T<T_{\text {eq }}$, além do que $d S /\left.d T\right|_{T_{\text {eq }}}=0$ conforme mencionado anteriormente. Logo a entropia total dos blocos é crescente em $T<T_{\text {eq }}$ até atingir um valor máximo em $T_{\text {eq }}$.

Para mostrar que a entropia atinge, de fato, um valor máximo no equilíbrio vamos calcular a derivada segunda da entropia. Da Eq. (8) temos

$$
\left.\frac{d^{2} S}{d T^{2}}\right|_{T_{\mathrm{eq}}}=-C_{\mathbf{A}}\left\{\frac{1}{T_{\mathrm{eq}}^{2}}+\frac{C_{\mathbf{A}} / C_{\mathbf{B}}}{\left[T_{\mathbf{B} i}-C_{\mathbf{A}} / C_{\mathbf{B}}\left(T_{\mathrm{eq}}-T_{\mathrm{A} i}\right)\right]^{2}}\right\} .
$$

que é estritamente negativa. Portanto a entropia assume um valor máximo após a termalização do sistema. Assim a variação de entropia dos blocos no referido processo é positiva $\left(\Delta S_{\text {sist }}>0\right)$, condizendo com a versão entrópica da segunda lei da termodinâmica.

\section{Agradecimentos}

Os autores agradecem ao Departamento de Física da Universidade Regional do Cariri. D.L. agradece ao programa PIBIC-URCA pelo apoio finaceiro. 


\section{Referências}

[1] F.M.S. Lima, Revista Brasileira de Ensino de Física 37, 1701 (2015).

[2] R. Resnick, D. Halliday e J. Walker Fundamentos de Física 2 (LTC, Rio de Janeiro, 2009), $8^{\mathrm{a}}$ ed.

[3] H.M. Nussenzveig, Curso de Física Básica 2 (Ed. Blucher, São Paulo, 2002), $4^{\mathrm{a}}$ ed.

[4] M.J. de Oliveira, Termodinâmica (Ed. Livr. da Física, São Paulo, 2012), $2^{\mathrm{a}}$ ed.

[5] E. Fermi, Thermodynamics (Dover Publ., New York, 1956).

[6] F.M.S. Lima, Eur. J. Phys. 36, 068001 (2015).

[7] G. Ávila, Cálculo 1 (LTC Ed., Rio de Janeiro, 2003), $7^{\mathrm{a}}$ ed., p. 208.

[8] N.A. Lemos, Convite à Física Matemática (Ed. Livr. da Física, São Paulo, 2013), p. 166.

[9] R. Resnick e D. Halliday, Física, Volume 2 (LTC, Rio de Janeiro, 1984), $4^{\mathrm{a}}$ ed. 